\title{
PRELIMINARY STUDIES FOR ALTERNATIVE LATTICE CORE DESIGN FOR FDM 3D PRINTED SANDWICH PANELS
}

\author{
Lam Man Chun ${ }^{1}$, Michał Kowalik ${ }^{1}$ \\ 1 Warsaw University of Technology, Institute of Aeronautics and Applied Mechanics, \\ Nowowiejska 24, 00-665 Warsaw, Poland. e-mail: mkowalik@meil.pw.edu.pl
}

\section{Introduction}

Lattice structures with different types of open cell topologies are attracting interest as millimetre cell sized lightweight core structures for sandwich panel constructions. Lattices appear to be an alternative improvement to honeycomb structures with flexible and amenable to the creation of singly or compound curved sandwich panels. Currently, lattice structures design is very limited to folding farication. Hence, Fused deposition modelling (FDM) 3D printing technologies allows more complex and advanced lattices design.

The purpose of this research is to design and compare capacity of different lattice structures. Z-ULTRAT material was used to simulate the 3D printed materials. All designs will undergo buckling test to simulate the real-world applications of sandwich core panels.

\section{Design Parameters}

\subsection{Unit Cell Densities}

For the design of lattice structure as shown in fig. 1, the unit cell density will be set to 0.3 while the height will be set to $10 \mathrm{~mm}$. From there, other dimensions of the lattice structures will be calculated according to Eq. (1). With the added weight of the material not taken account; only the cross sectional area and length determine the relative density of the structures. The unit cell are arranged in an array pattern to contain in $50 \mathrm{~mm} x$ $50 \mathrm{~mm}$ area.

$$
\tilde{p}=\frac{\text { Volume of Truss }}{\text { Volume of Unit Cell }}
$$

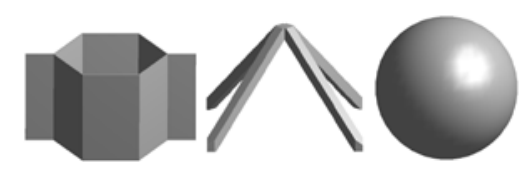

Fig. 1. Lattices Unit Cell Design of Honeycomb, Pyramidal and Hollow Sphere shape.

\section{Compression Test}

\subsection{Testing layout}

The models are simulated using ANSYS 16.0 and tested via INSTRON Testing system. The sandwich core is placed between two $7 \mathrm{~cm}$ solid steel cylinder. Load is applied from the bottom while the fixture is set at the top of the sandwich core as shown in fig. 2. The INSTRON Testing system is set to compress $4 \mathrm{~mm}$ to the core design.

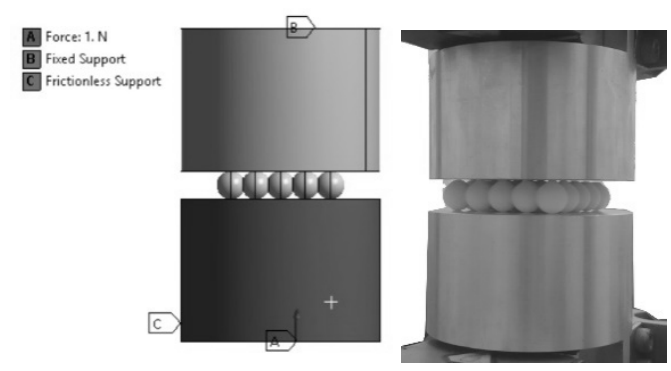

Fig. 2. Compression Test of Sandwich Panel. FE model (left), experimental setup (right).

\subsection{Testing Material}

The material used for the buckling simulation is Z-ULTRAT from Zortrax to simulate the commonly used 3D printing thermoplastics.

\begin{tabular}{|c|c|}
\hline Parameters & Z-ULTRAT \\
\hline Mass Density [g/cm ${ }^{3}$ ] & 1.04 \\
\hline Young Modulus [MPa] & 857 \\
\hline Poisson's Ratio & 0.36 \\
\hline
\end{tabular}

Table 1. Properties of the testing materials

\section{FDM 3D Printing Parameters}

The lattice design will be printed by using fused deposition modelling (FDM) 3D printing technology. The models will be slice by Zortrax ZSuite software with a resolution of 100 microns and print out by Zortrax M200. The Z-ULTRAT materials is melted and printed at a temperature of $270^{\circ} \mathrm{C}$. 


\section{Results}

Figure 3 shows the result from the Buckling test ANSYS Workbench 16.0 simulated and the Compression test result. Table 2 shows the time and amount of filament required to print out each of the respective core design models.

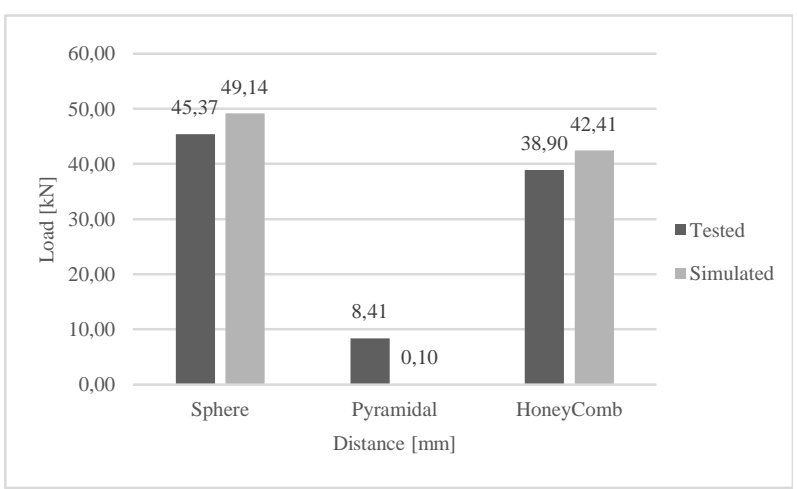

Fig. 3. Buckling Result of Core design

\begin{tabular}{|c|c|c|}
\hline Core Design & $\begin{array}{c}\text { Build Time } \\
\text { [Hours] }\end{array}$ & $\begin{array}{c}\text { Filament Used } \\
{[\mathbf{m m}]}\end{array}$ \\
\hline Honeycomb & 2.67 & 2248.4 \\
\hline Pyramidal & 5.38 & 3231.2 \\
\hline Hollow Sphere & 3.75 & 3387.6 \\
\hline
\end{tabular}

Table 2. Properties of the testing materials

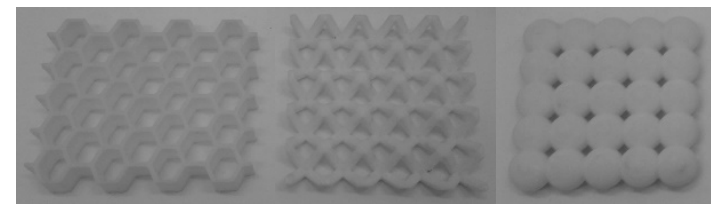

Fig. 4. 3D Printed Model of Honeycomb, Pyramidal and Sphere samples.

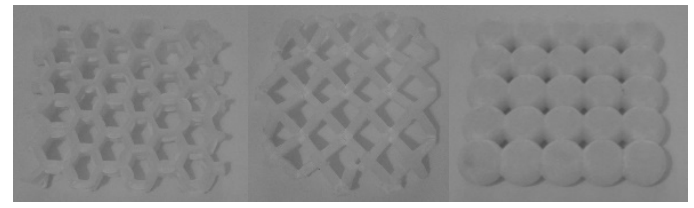

Fig. 5. Result of Compressed Honeycomb, Pyramidal and Sphere samples.

\section{Conclusions}

From initial simulation result, Spherical lattice design has the highest critical buckling load at a given height of $10 \mathrm{~mm}$ among the 3 lattices designs.

Spherical lattice design also required the second least time but more amount of filament to print. Honeycomb core design has the lower buckling load but used the least filaments and time to print out the test specimen.

Pyramidal has the longest build time and used the most filament and yet to achieve the lowest critical buckling load. This is because the Pyramidal design is not bonded to a fixed surface at the top and bottom, causing the pyramidal break at the joints of the legs and spilt the legs apart as shown in figure 6.

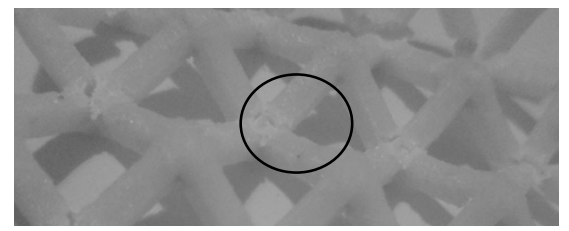

Fig. 6. Fault at Pyramidal Core Design

\section{Remarks}

- Data is created by the simulations has yet to be fully analyzed, therefore only initial findings are reported here.

- The time and amount of filament used to print the core designs varies among different types of 3D printers as they have different calibrations and specifications.

\section{References}

[1] Douglas T. Queheillalt, Haydn N.G. Wadley, Pyramidal Lattice Truss Structures with hollow Trusses, 2005

[2] Li Yang, Ola Harrysson, Harvey West, Denis Cormier, A comparison of Bending Properties for Cellular Core Sandwich Panels, 2013.

[3] Haydn N.G. Wadley, Multifunctional Periodic Cellular Metals, 2005

[4] Douglas T. Queheillalt,Yellapu Murty, Daydn N.G. Wadley, Mechanical Properties of an extruded pyramidal lattice truss sandwich structure, 2007.

[5] Brooks W.K, Todd J. and Sutcliffe C.J., The Production of Open Cellular Lattice Structures Using Selective Laser Melting, In: Sixth National Conference on Rapid Prototyping, Design, and Manufacturing, 2005 